\title{
Structural characterization of seven-bladed beta-propeller lectin family from Photorhabdus laumondii
}

\author{
E. Fujdiarová ${ }^{1,2}$, J. Houser ${ }^{1,2}$, F. Melicher ${ }^{1}$, M. Wimmerová,, ${ }^{1,23}$ \\ ${ }^{1}$ National Centre for Biomolecular Research, Kamenice 5, Brno 625 00, Czech Republic, \\ ${ }^{2}$ Central European Institute of Technology, Kamenice 5, Brno 625 00, Czech Republic, \\ ${ }^{3}$ Department of Biochemistry, Faculty of Science, Masaryk University, Kotlařská 2, Brno 611 37, Czech Republic \\ eva.fujdiarova@mail.muni.cz
}

Lectins, carbohydrate recognizing proteins, play an important role in various physiological and pathophysiological processes as well as both mutualistic and parasitic interactions between microorganisms and hosts [1]. In connection with the last-mentioned process, lectins from pathogenic bacteria can mediate the first step of infection and they are considered an important virulence factor.

Photorhabdus laumondii is an entomopathogenic bacterium, which is known for its complicated life cycle, including mutualism and pathogenicity towards two different invertebrate hosts [2]. This contribution is focused on the newly described PLL lectin family, which shares a seven-bladed beta-propeller fold. All five members of this family are highly similar to each other in primary, secondary, and tertiary structure. However, the oligomeric state of these lectins differs significantly. Members of the PLL family have been confirmed to bind multiple monosaccharides, including L-fucose and $O$-methylated saccharides. X-ray structures of PLL family discovered two sets of binding sites with different ligand specificity per monomer, "polar" sites and "hydrophobic" sites. Amino acids involved in the ligand-binding are highly conserved within the lectin molecule. Ligands are bound in both types of binding sites via hydrogen bonds and via CH- $\pi$ interaction with aromatic residues. Lectin/saccharide interaction is mostly mediated via hydrogen bonds. However, hydrophobic sites are deepened with a hydrophobic pocket. The importance of non-polar interactions, such as $\mathrm{CH}-\pi$ interactions between aromatic amino acids and apolar part of carbohydrate molecules, was shown recently [3].

[1] Lis, H. and Sharon, N. (1998) Chem Rev. 98, 637-74.

[2] Clarke, DJ. (2020) Microbiology. 166, 335-348.

[3] Wimmerová, M., Kozmon, S., Nečasová, I., Mishra, S.K., Komárek, J., Koča, J. (2012) Plos One. e46023.

\section{Keywords: beta-propeller; lectin; Photorhabdus}

The authors would like to acknowledge the BESSY II electron storage ring (Berlin-Adlerhof, Germany) and DESY, the member of the Helmholtz Asociation HGF (Hamburg, Germany), for access to the synchrotron data collection facility and allocation of synchrotron radiation beam time. CIISB research infrastructure project LM2018127 funded by MEYS CR is gratefully acknowledged for the financial support of the measurements at the CF Biomolecular Interactions and Crystallization, CEITEC MU. This work was supported by GAČR (21-29622S). 